\title{
Smoking and stress in the general population in Denmark
}

\author{
Lone P. Buhelt', Charlotta Pisinger ${ }^{1,2,3}$, Anne H. Andreasen ${ }^{1}$
}

\begin{abstract}
INTRODUCTION The social pressure placed on smokers today might potentially lead to an increasing level of stress. We investigated if the proportion of persons with high stress level had increased over time more in smokers than in non-smokers. METHODS Data were obtained from repeated cross-sectional surveys of The Capital Region Health Survey conducted in 2010, 2013 and 2017. Survey data were weighted for survey design and non-response, and linked to national register data. Cohens Perceived Stress Scale (PSS-10) score was used. Logistic regression analyses, based on 136608 citizens' self-reports, were adjusted for sex, age, education level, employment, and alcohol intake (and loneliness, in analysis investigating the associations between tobacco consumption and high stress level).

RESULTS A significantly higher proportion of citizens reported a high stress level in 2017 compared with 2010 and 2013 but there was not a greater increase in smokers than in non-smokers. Daily smoking men had $69 \%$ higher odds of reporting perceived high stress level and daily smoking women had 36\% higher odds, than never smokers of the same sex. There was a significant trend between higher daily tobacco consumption and a higher proportion of smokers with high stress level.

CONCLUSIONS The increase in high stress level over time occurred independently of smoking status. Daily smokers had the highest odds of perceived high stress level, and a higher daily tobacco consumption was associated with a higher proportion of smokers with high stress level. Smoking cessation programs should, to a higher degree, consider implementing stress-coping elements to prevent relapse.
\end{abstract}

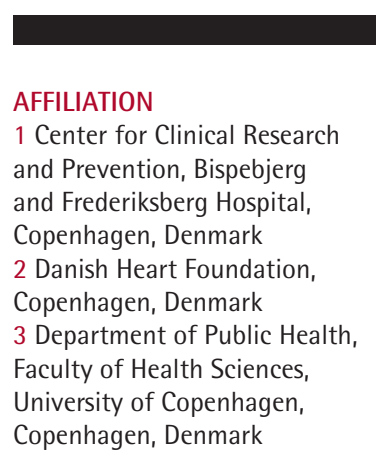

CORRESPONDENCE TO

Lone P. Buhelt. Center for Clinical Research and Prevention, Bispebjerg and Frederiksberg Hospital, Hovedvejen, Nordre Fasanvej 57, DK-2000, Copenhagen, Denmark. E-mail: lonebuhelt@ outlook.dk

\section{KEYWORDS}

smoking, stress, psychological, mental health, population-based, Denmark

Received: 1 September 2020 Revised: 22 January 2021

Accepted: 22 January 2021

\section{INTRODUCTION}

The epidemic of disease caused by smoking ranks among the greatest public health catastrophes of the last century ${ }^{1-3}$. Despite this knowledge, smoking is still the leading preventable cause of death in the Western world ${ }^{1}$. In Denmark, the smoking prevalence has steadily decreased over many decades but has stagnated for seven years on a daily smoking rate of $17 \%{ }^{4}$. Furthermore, the smoking prevalence among the young adults has increased in 2017, which is alarming ${ }^{4}$.

The health consequences of smoking have led to a range of public health initiatives. High cigarette taxes, smoke-free legislation, comprehensive marketing bans, media campaigns, smoking cessation treatment/clinics, and graphic health warnings have important roles in reducing the smoking prevalence ${ }^{5}$. A large body of evidence of the benefits of tobacco control exist, but the potential unintended consequences of this public health strategy have received little attention. Smokers report perceiving smoke-free legislation as increasing the stigmatization of smoking ${ }^{6}$. The social pressure placed on smokers might potentially lead to an increasing level of stress and social isolation, a feeling of increased resistance to quitting smoking and to increased relapse ${ }^{7}$. A recent experimental study showed that stigmatization led smokers toward emotional, cognitive, and attitudinal reactions that 
might make them less likely to quit smoking ${ }^{8}$.

Smokers in the general population have been found to have higher stress hormone levels than nonsmokers ${ }^{9}$. Prospective studies have shown that a high stress level is associated with nearly double the odds of failure to quit smoking ${ }^{10}$ and experimental studies have shown that following a stressful event, smokers experienced increased tobacco craving, smoked more intensely and were less able to resist smoking ${ }^{11}$.

Large repeated cross-sectional surveys in the Capital Region of Denmark give us a unique opportunity to investigate the association between smoking and stress, at population level. To our knowledge, no previous studies have investigated the temporal changes in stress in smokers and nonsmokers, at population level.

We hypothesized that, if tobacco control policies have increased the stigmatization of smokers, we would see a greater increase in stress in smokers than in non-smokers.

The aim was to investigate if Danish smokers in the general population reported high stress levels more frequently than non-smokers, if there has been an increase during the period 2010-2017 in citizens' perceived high stress, and if the proportion of persons with high stress levels has increased more in smokers than in non-smokers. Finally, we wanted to describe the association between tobacco consumption and high stress level in daily smokers.

\section{METHODS}

The Capital Region, consisting of 29 municipalities, is the largest region of Denmark, with approximately 1.5 million inhabitants aged $\geq 16$ years. Data were obtained (on paper or online) from repeated crosssectional surveys of The Capital Region Health Survey conducted in 2010, 2013 and 2017. Random samples of all citizens were drawn from the Civil Registration System using computer-generated random numbers. Due to differences in population size among municipalities, one municipality increased the sample size to 4500 individuals, and Copenhagen Municipality was divided into ten areas, which were treated as individual municipalities in the sampling process. Each municipality drew a random sample of at least 2450 individuals ( $\geq 16$ years). In 2017 , the sample was increased to 4900 in four municipalities. Two to four reminders were sent.
The first survey, conducted in 2010, included a total sample of 95150 invited individuals. The final sample consisted of 49806 individuals $(52.3 \%$ response rate). The second survey, conducted in 2013, included a total sample of 95150 invited individuals. The final sample consisted of 41356 individuals (43.5\% response rate). The third survey, conducted in 2017, comprised a sample of 104950 invited individuals. The final sample consisted of 55185 individuals ( $52.6 \%$ response rate).

All citizens in Denmark are given a unique and permanent personal identification number at birth or on immigration, which allowed us to link data to national register data at the individual level.

The project was approved by the Danish Data Protection Agency. Approval from the Danish Health Research Ethics Committee System was not required according to Danish law. Written informed consent for publication of data was obtained.

\section{Variables}

Stress (self-reported)

Cohens Perceived Stress Scale (PSS-10) score was used ${ }^{12}$. PSS-10 consists of 10 items regarding predictability, controllability and life-overload, as perceived by the respondents during the past four weeks (range: 0-40). The higher the score, the greater the feeling of psychological stress. The scale has been validated and the Danish consensus version of the PSS-10 has good psychometric properties ${ }^{13}$. The PSS10 was dichotomized into high stress level (PSS-10 $\geq 18$ ) corresponding to the $20 \%$ with the highest score among respondents in 2010 and medium/low stress level (PSS-10<18).

\section{Smoking status (self-reported)}

Smoking status was classified as: daily smoker, occasional smoker ('yes, minimum once a week' or 'yes, less often than every week'), ex-smoker, and never smoker.

\section{Tobacco consumption (self-reported)}

Tobacco consumption was classified as: one cigarette/ gram of pipe tobacco $=1 \mathrm{~g}$, one cheroot $=3 \mathrm{~g}$, and one cigar $=5 \mathrm{~g}$ of tobacco.

\section{Alcohol consumption (self-reported)}

This was ascertained by the following questions: 
'Have you been drinking alcohol the last 12 months?' and 'How much alcohol have you typically been drinking every day during the week?'. The respondents were dichotomized into 'as recommended' (0-14 units per week for women and 0-21 units per week for men) and 'above recommendation', based on the recommendations of the Danish Health Authorities.

\section{Loneliness (self-reported)}

A Danish version of the Three-Item Loneliness Scale ${ }^{14}$ was used based on the following questions: 'How often do you feel that you lack companionship?'; 'How often do you feel left out?'; and 'How often do you feel isolated from others?'. Response was evaluated on a 3-point Likert scale (hardly ever $=1$, sometimes $=2$, often $=3$ ). Higher scores indicate greater degrees of loneliness. Subsequently, the respondents were dichotomized into 'being lonely' (total score $=7-9$ ) or 'not lonely' (total score $<7$ ). The scale is based on the UCLA Loneliness Scale ${ }^{15}$, the gold standard measure in loneliness research.

\section{Sociodemographic characteristics}

Age, gender, educational level, cohabitation status and employment status were obtained from national registers at Statistics Denmark. Education and employment were used as indicators of socioeconomic position. The educational level was categorized into four groups based on the individuals' highest level of completed education: 'Basic/student' (primary or secondary school only/under education); 'Low education' (up to two years of vocational training, e.g. carpenter, hairdresser); 'Medium education' (two to four years of education e.g. teacher, nurse); and 'High education' (four years or more of academic education, e.g. doctor, psychologist).

Employment data were obtained from the Income Statistics Register. Employment status was categorized as: 'Employed/under education'; 'Unemployed' (unemployed, long-term ill, on disability pension); or 'Retired'.

\section{Cohabitation status}

This was dichotomized into 'Yes' (live with partner) and 'No' (live alone). Persons aged 16-24 years living with their parent(s) were registered with their parent(s) cohabitation status.

\section{Statistical analysis}

All analyses were weighted for survey design and non-response (higher among men, persons who were younger, had a lower income, were living alone, were unemployed, or/and were of an ethnicity other than Danish). The survey weights were computed by Statistics Denmark based on registry information about sex, age, municipality of residence, highest completed education level, income, civil status and hospitalization, for all individuals invited to the survey.

Descriptive results are shown as number of respondents and weighted percentage. Logistic regression analyses were used to investigate whether there were changes from 2010 to 2017 in selfreported high stress level, adjusting for sex, age, education level, employment and alcohol intake, and whether these changes differed with sex and across smoking status.

Logistic regression was used to investigate the associations between smoking status and high stress level using data from 2017, since information about loneliness was only available in 2017 . The analyses were adjusted first for sex and age and then further adjusted for education level, employment, alcohol consumption, and loneliness.

Nine categories for tobacco consumption (1-5, 6-10, 11-15, 16-20, 21-25, 26-30, 31-35, 36-40, $\geq 40$ g) were created among daily smokers in 2017 . The percentage of persons with high stress level was calculated within each of these categories. A linear regression was used to estimate the linear trend between tobacco consumption and high stress level. Statistical analyses were performed using SAS statistical software (version 9.4, SAS Institute Inc., Cary, NC, US).

\section{RESULTS}

The total number of respondents was 146347 . Of these, 136608 reported both smoking status and PSS10 and were included in this study. Characteristics of the population are given in Table 1, where approximately half of the respondents were aged 3564 years, there were slightly more women, and four in ten had a medium or high education. Three in four were employed and six in ten lived with a partner. In 2010, the median perceived stress score (PSS-10) was 11.4 (IQR: $7.0-16.3$ ) and $22.1 \%$ reported a high 
stress level. In 2013, the median score was 10.5 (IQR: $5.9-15.8)$ and $20.6 \%$ reported a high stress level. In 2017, the median score was 11.5 (IQR: 6.5-17.1) and $25.5 \%$ reported a high stress level, and $16 \%$ were daily smokers ( $1 \%$ point lower than the national smoking rate). Daily smoking had decreased while occasional smoking increased over the seven years.

In adjusted analyses, we investigated the temporal changes in citizens' reporting of perceived high stress level. We found a significant increase over time ( $\mathrm{OR}=1.25$; 95\% CI: 1.20-1.30) for 2017 compared to $2010(p<0.0001)$. The increase did not differ

Table 1. Characteristics of the study population in Capital Region of Denmark in 2010, 2013 and 2017. Only persons with information on smoking and stress are included

\begin{tabular}{|c|c|c|c|c|c|c|}
\hline \multirow[t]{2}{*}{ Characteristics } & \multicolumn{2}{|c|}{2010} & \multicolumn{2}{|c|}{2013} & \multicolumn{2}{|c|}{2017} \\
\hline & $n *$ & $\% * *$ & $n *$ & $\% * *$ & $n *$ & $\% * *$ \\
\hline \multicolumn{7}{|l|}{ Sex } \\
\hline Female & 25909 & 51.3 & 21228 & 51.4 & 28679 & 51.2 \\
\hline Male & 20391 & 48.7 & 16671 & 48.6 & 23730 & 48.8 \\
\hline \multicolumn{7}{|l|}{ Age (years) } \\
\hline $16-34$ & 10767 & 31.8 & 8204 & 32.4 & 11801 & 33.2 \\
\hline $35-64$ & 25601 & 50.7 & 19538 & 49.1 & 26731 & 47.2 \\
\hline$\geq 65$ & 9932 & 17.5 & 10157 & 18.6 & 13877 & 19.6 \\
\hline \multicolumn{7}{|l|}{ Education level } \\
\hline Basic/student & 13836 & 35.0 & 10545 & 33.9 & 14295 & 34.0 \\
\hline Low & 14239 & 28.2 & 11221 & 26.4 & 15343 & 25.0 \\
\hline Medium & 11313 & 23.4 & 9814 & 24.5 & 13650 & 24.3 \\
\hline High & 6059 & 13.4 & 5695 & 15.3 & 8377 & 16.7 \\
\hline \multicolumn{7}{|l|}{ Employment } \\
\hline Employed/under education & 32431 & 73.0 & 25310 & 72.4 & 35815 & 72.7 \\
\hline Unemployed & 2632 & 7.8 & 2107 & 8.0 & 3081 & 8.4 \\
\hline Retired & 10235 & 19.2 & 9842 & 19.6 & 12088 & 18.8 \\
\hline \multicolumn{7}{|l|}{ Cohabitation (age $\geq 25$ years) } \\
\hline Live with a partner & 29415 & 62.8 & 24328 & 60.8 & 32568 & 60.7 \\
\hline Live alone & 12011 & 37.2 & 9643 & 39.2 & 14556 & 39.3 \\
\hline \multicolumn{7}{|l|}{ Smoking status } \\
\hline Daily smoker & 8389 & 19.7 & 5229 & 15.5 & 7376 & 16.0 \\
\hline Occasional smoker & 2175 & 5.7 & 1759 & 6.1 & 2962 & 7.3 \\
\hline Ex-smoker & 14810 & 28.9 & 12772 & 29.4 & 17452 & 29.0 \\
\hline Never smoker & 20926 & 45.7 & 18139 & 49.0 & 24619 & 47.6 \\
\hline \multicolumn{7}{|l|}{ Perceived stress (PSS-10) } \\
\hline Low/medium stress & 37200 & 77.9 & 31074 & 79.4 & 40625 & 74.5 \\
\hline High stress & 9100 & 22.1 & 6825 & 20.6 & 11784 & 25.5 \\
\hline \multicolumn{7}{|l|}{ Alcohol consumption } \\
\hline As recommended & 39308 & 87.1 & 32948 & 90.2 & 46469 & 91.6 \\
\hline Above recommendation & 5690 & 12.9 & 3640 & 9.8 & 4284 & 8.4 \\
\hline \multicolumn{7}{|l|}{ Loneliness } \\
\hline Not lonely & . & 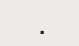 & . & . & 48676 & 91.8 \\
\hline Lonely & . & . & . & . & 3215 & 8.25 \\
\hline
\end{tabular}

* Number of respondents with information on smoking and stress score. ${ }^{* *}$ Weighted for design and non-response. 
between men and women $(\mathrm{p}=0.680)$ and there was no significant difference in temporal development between the four smoking status groups $(p=0.055)$.

In logistic regression models, we investigated the association between smoking status and perceived high stress level. Analyses showed a significant interaction between smoking and $\operatorname{sex}(\mathrm{p}<0.0001$ adjusted for age, $p=0.0005$ fully adjusted) and results are therefore shown by sex (Table 2). We found a significant association between smoking status and perceived high stress level, except that there was no significant difference between exsmoking and never smoking women. Adjusted odds ratio for daily smoking men was 2.16 (95\% CI: 1.93-2.41) compared to never smoking men. Similar results but less pronounced were seen among women. Also, for both sexes, we found that never smokers had the lowest probability of reporting perceived high stress level, followed by ex-smokers and occasional smokers. Daily smokers had the highest probability. In the fully adjusted models, odds ratios decreased, especially for daily smokers. Daily smoking men had 69\% higher odds of reporting perceived high stress level and daily smoking women had $36 \%$ higher odds, than never
Table 2. Smokers' and non-smokers' probability of having a high stress level, by sex, 2017

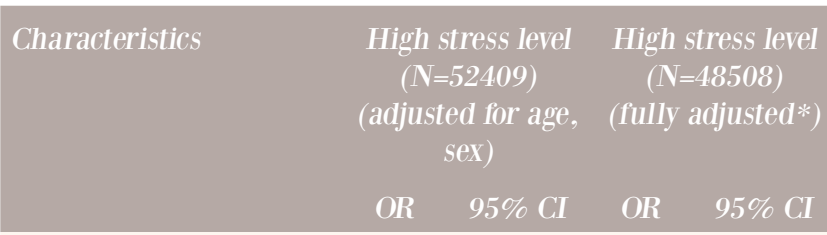

Men

Never smokers 1

Ex-smokers $\begin{array}{llll}1.43 & 1.29-1.57 & 1.34 & 1.21-1.49\end{array}$

Occasional smokers

$1.37 \quad 1.17-1.61 \quad 1.38 \quad 1.15-1.64$

Daily smokers

$2.16 \quad 1.93-2.41 \quad 1.69 \quad 1.50-1.91$

Women

Never smokers

Ex-smokers

Occasional smokers

$\begin{array}{llll}1.06 & 0.99-1.14 & 1.03 & 0.95-1.12\end{array}$

Daily smokers

$1.34 \quad 1.17-1.55 \quad 1.29 \quad 1.10-1.51$

$1.78 \quad 1.62-1.96 \quad 1.36 \quad 1.22-1.51$

*Adjusted for sex, age, education level, employment, alcohol, and loneliness.

smokers of same sex.

Finally, we investigated daily smokers' tobacco consumption and perceived stress level (Figure 1), based on 7376 observations. We found that when

Figure 1. The association between tobacco consumption and perceived high stress level in 7376 daily smoking citizens in the Capital Region of Denmark in 2017

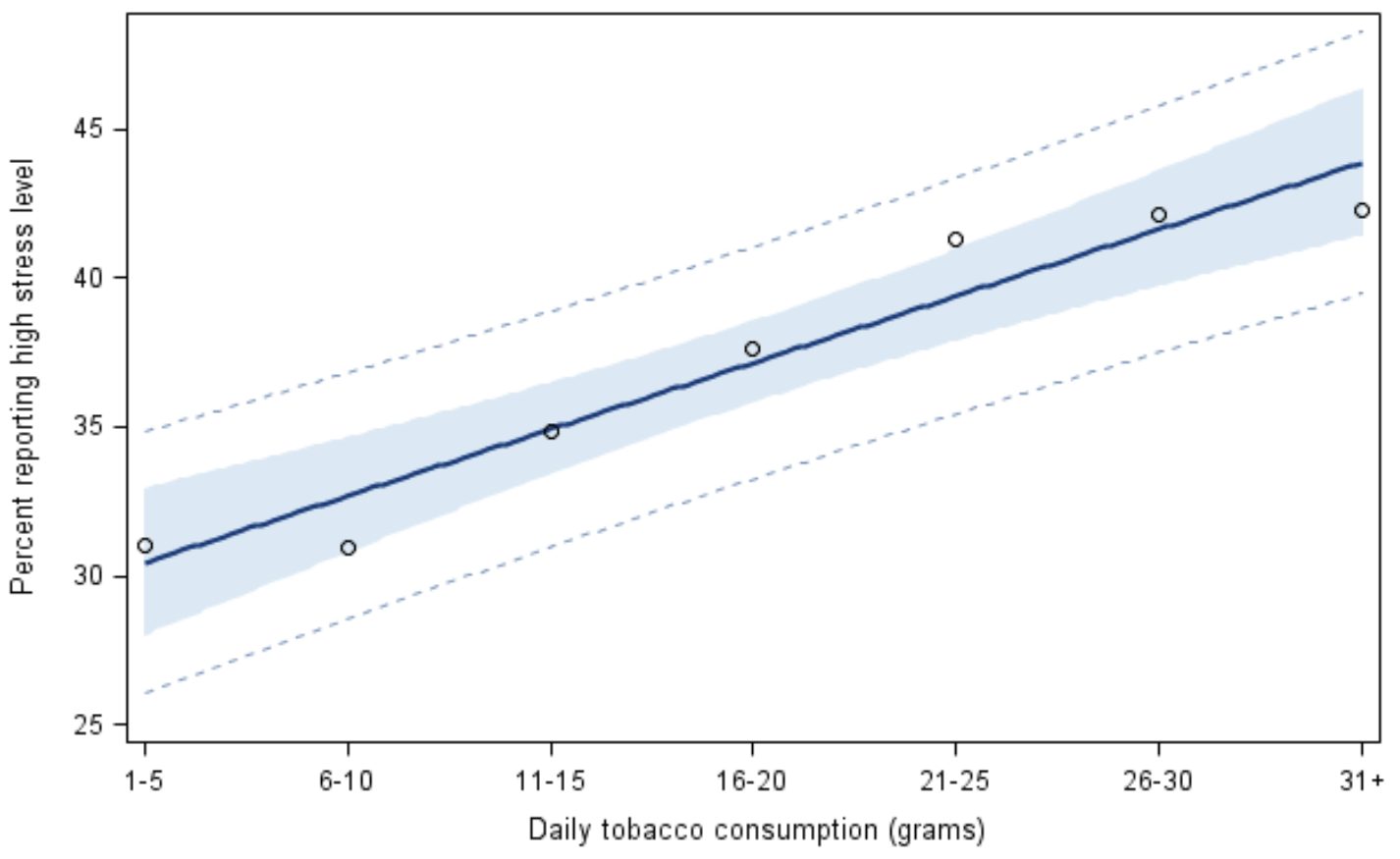

Linear regression analyses 
tobacco consumption increased, a higher proportion of persons reported a perceived high stress level. Test for trend was significant $(\mathrm{p}=0.0004)$. In all, $40.3 \%$ of men and $44.6 \%$ of women with a tobacco consumption of 21-26 cigarettes/g tobacco per day had a perceived high stress level.

\section{DISCUSSION}

In repeated cross-sectional population-based surveys in the Capital Region of Denmark, we found a significantly increased proportion of citizens with perceived high stress level in 2017 compared with 2010 and 2013. There was no difference in increase in perceived high stress level between smokers and nonsmokers. In analyses adjusted for sociodemographic factors, alcohol intake and loneliness, daily smokers had the highest odds of perceived high stress level, followed by occasional smokers and ex-smokers. Never smokers had the lowest odds of perceived high stress level. Furthermore, we found that higher daily tobacco consumption was associated with a higher proportion of citizens with high stress level.

Smoke-free legislation, anti-smoking campaigns and social denormalization of tobacco use are successful tools to combat the devastating health effects of smoking but the social pressure placed on smokers might have negative side-effects. As smokers report that they have perceived the smokefree legislation to have increased the stigmatization of smoking ${ }^{16}$, we hypothesized that smokers might have become more stressed over time than nonsmokers. This could not be confirmed. To our knowledge, this is the first study to have investigated the temporal changes in stress in smokers and nonsmokers, at population level.

The association between smoking and stress is complex. The question about causality remains, but studies indicate that there is a bidirectional association.

Prospective studies indicate that stress in childhood/adolescents is a causal risk factor of smoking initiation. A large cohort study followed children for 16 years and found that girls with high stress at age three had over two times higher odds than girls with low stress to start smoking early ${ }^{16}$. Another large cohort study showed that low stress resilience in late adolescence increased the risk of smoking in adulthood ${ }^{17}$. A population- based cross-sectional study, measuring stress with the same tool as our study, found that a one-unit increase in perceived stress was associated with in a significant $5 \%$ increased odds of smoking ${ }^{18}$. Also, those with high stress levels, such as citizens with post-traumatic-stress-disorder (PTSD) have very high smoking rates ${ }^{19}$ and stress seems to increase smoking desire, in established smokers. Smokers report that stress relief is one of the main reasons for smoking ${ }^{20}$. Experimental human studies have shown that cigarette craving was increased after stress exposure ${ }^{21,22}$ and was related to the magnitude of cortisol stress response ${ }^{21}$. Animal studies confirm that stress during abstinence periods induces selfadministration of nicotine intake ${ }^{23}$. There is a strong socioeconomic gradient in smoking in many countries, and chronic financial stress has been found to partly explain the socioeconomic gradient in smoking ${ }^{24}$.

Evidence also indicates that perceived stress is a barrier for smoking cessation. Persons with high level of stress (PTSD) have lower odds of quitting ${ }^{25}$ and in prospective studies of smokers enrolled in a smoking cessation program, stress predicted relapse ${ }^{26}$.

On the other hand, nicotine abstinences are stressful and nicotine exposure seems to increase stress levels and exacerbate mood disturbances by inducing changes in neural pathways and neurotransmitter systems that are implicated in mood regulation ${ }^{27}$. A recent review found evidence for smoking being associated with subsequent depression and anxiety and vice versa ${ }^{28}$.

Finally, studies have studied stress levels after smoking cessation. In our study, we found that exsmokers had much lower odds of perceived high stress levels than daily smokers, but not as low as never smokers. Previous studies confirm that smoking cessation is associated with decreased, not increased stress ${ }^{29,30}$, even in highly dependent smokers who reported that smoking helped them cope with stress ${ }^{29}$. A systematic review, based on 23 studies, concluded that smoking cessation was associated with a decrease in stress levels and an increase in positive mood compared to continuing smoking ${ }^{30}$. Noteworthy, a study has shown that those who tried to quit smoking but failed had higher odds of stress than those who did not try to quit ${ }^{31}$. 
In our study, we found a dose-response association between smoking intensity and perceived high stress level, which confirms previous findings. A large cross-sectional study showed that increasing levels of perceived stress were significantly associated with heavy smoking among daily smokers ${ }^{18}$, and a large cohort study found that higher work stress level was associated with greater smoking intensity among smokers $^{32}$.

\section{Strengths and limitations}

The large randomly selected population-based sample, a broad age span, together with use of a weighting procedure ensured a high degree of national generalizability. Measurement of stress was done by use of a validated widely used scale and we adjusted for several potential confounders such as education level, loneliness, and alcohol use.

The study has limitations. First, the most important weakness is the cross-sectional study design, which implies that no conclusions on causality between smoking and stress can be drawn. Second, the chosen stress-scale does not necessarily cover all aspects of stress. A study indicated scalability problems in the current Danish version of PSS-10 $0^{33}$. Also, the PSS-10 questionnaire relies on the respondent's retrospective memory in the last four weeks, imposing a risk of recall bias. Dichotomizing a variable has weaknesses; e.g. a person who just missed the cut-off point of being among the $20 \%$ of persons with the highest stress level is classified as not-stressed. Further, data on smoking, alcohol, loneliness and stress are self-reported, so there is risk of information bias. Selection bias cannot be ruled out even though the population weights were included to compensate for non-response.

Smoke-free legislation was implemented in Denmark already in 2007 and since then there has only been a small tightening of the smoke-free environment Act and of other tobacco control Acts. However, many municipalities, workplaces and schools have voluntarily implemented much stronger smoking bans. Further, because of the stagnation of the smoking prevalence in Denmark there has been an increasing public and political focus on smoking, its negative health effects and the need for a stronger tobacco control. We believe, that there has been significantly increased social pressure on smokers from 2010 to 2017.

\section{CONCLUSIONS}

In two cross-sectional large population-based studies we found that the proportion of citizens with perceived high stress level had increased over time, but there was no difference in increase between smokers and non-smokers. Daily smokers had the highest odds of perceived high stress level, even when we adjusted for confounders, and a higher daily tobacco consumption was associated with a higher proportion of citizens with perceived high stress level.

Smoke-free legislation, anti-smoking campaigns and social denormalization of tobacco have proven successful in reducing smoking in the population and are important elements of tobacco control. Even though our study did not find that the increase in stress was steeper in smokers than in non-smokers, indicating that the tobacco control strategies have not stressed smokers, we found a worryingly high proportion of daily smokers with perceived high stress level. As a high stress level seems to increase the resistance to quitting and the risk of relapse during a smoking cessation attempt, smoking cessation programs should, to a higher degree, consider implementing stress-coping elements to prevent relapse, especially in heavy smokers. Mindfulness has shown to be a promising intervention to reduce stress and improve cessation outcomes, among smokers with low socioeconomic status $^{34}$.

\section{REFERENCES}

1. Drope J, Schluger N, Cahn Z, et al. The Tobacco Atlas. Atlanta, GA: American Cancer Society, Vital Strategies; 2018. https://tobaccoatlas.org/wp-content/ uploads/2018/03/TobaccoAtlas_6thEdition_LoRes_ Rev0318.pdf. Accessed January 22, 2021.

2. U.S. Department of Health and Human Services. How Tobacco Smoke Causes Disease: The Biology and Behavioral Basis for Smoking-Attributable Disease: A Report of the Surgeon General. Atlanta, GA: U.S. Department of Health and Human Services, Centers for Disease Control and Prevention, National Center for Chronic Disease Prevention and Health Promotion, Office on Smoking and Health; 2010. https://www.ncbi.nlm.nih. gov/books/NBK53017/pdf/Bookshelf_NBK53017.pdf. Accessed January 22, 2021.

3. U.S. Department of Health and Human Services. The 
Health Consequences of Smoking: 50 Years of Progress. A Report of the Surgeon General. Atlanta, GA: U.S. Department of Health and Human Services, Centers for Disease Control and Prevention, National Center for Chronic Disease Prevention and Health Promotion, Office on Smoking and Health; 2014. https://www.ncbi.nlm.nih. gov/books/NBK179276/pdf/Bookshelf_NBK179276. pdf. Accessed January 22, 2021.

4. Jensen HAR, Davidsen M, Ekholm O, Christensen AI. Danskernes Sundhed: Den Nationale Sundhedsprofil 2017 [in Danish]. Copenhagen, Denmark: Danish Health Authority; 2018. https://www.sst.dk/da/ udgivelser/2018/danskernes-sundhed-den-nationalesundhedsprofil-2017. Accessed April 4, 2021.

5. Levy DT, Tam J, Kuo C, Fong GT, Chaloupka F. The Impact of Implementing Tobacco Control Policies: The 2017 Tobacco Control Policy Scorecard. J Public Health Manag Pract. 2018;24(5):448-457. doi:10.1097/PHH.0000000000000780

6. Ritchie D, Amos A, Martin C. 'But it just has that sort of feel about it, a leper'--stigma, smoke-free legislation and public health. Nicotine Tob Res. 2010;12(6):622-629. doi:10.1093/ntr/ntq058

7. Evans-Polce RJ, Castaldelli-Maia JM, Schomerus G, EvansLacko SE. The downside of tobacco control? Smoking and self-stigma: A systematic review. Soc Sci Med. 2015;145:26-34. doi:10.1016/j.socscimed.2015.09.026

8. Helweg-Larsen M, Sorgen LJ, Pisinger C. Does it help smokers if we stigmatize them? A test of the stigmainduced identity threat model among U.S. and Danish smokers. Soc Cogn. 2019;37(3):294-313. doi:10.1521/ soco.2019.37.3.294

9. Wosu AC, Gelaye B, Valdimarsdóttir U, et al. Hair cortisol in relation to sociodemographic and lifestyle characteristics in a multiethnic US sample. Ann Epidemiol. 2015;25(2):9095.e2. doi:10.1016/j.annepidem.2014.11.022

10. Slopen N, Kontos EZ, Ryff CD, Ayanian JZ, Albert MA, Williams DR. Psychosocial stress and cigarette smoking persistence, cessation, and relapse over 9-10 years: a prospective study of middle-aged adults in the United States. Cancer Causes Control. 2013;24(10):1849-1863. doi:10.1007/s10552-013-0262-5

11. McKee SA, Sinha R, Weinberger AH, et al. Stress decreases the ability to resist smoking and potentiates smoking intensity and reward. J Psychopharmacol. 2011;25(4):490-502. doi:10.1177/0269881110376694

12. Cohen S, Kamarck T, Mermelstein R. A global measure of perceived stress. J Health Soc Behav. 1983;24(4):385396. doi:10.2307/2136404

13. Eskildsen A, Dalgaard VL, Nielsen KJ, et al. Crosscultural adaptation and validation of the Danish consensus version of the 10-item Perceived Stress Scale. Scand J Work Environ Health. 2015;41(5):486-490. doi:10.5271/sjweh.3510

14. Hughes ME, Waite LJ, Hawkley LC, Cacioppo JT. A
Short Scale for Measuring Loneliness in Large Surveys: Results From Two Population-Based Studies. Res Aging. 2004;26(6):655-672. doi:10.1177/0164027504268574

15. Russell DW. UCLA Loneliness Scale (Version 3): reliability, validity, and factor structure. J Pers Assess. 1996;66(1):20-40. doi:10.1207/s15327752jpa6601_2

16. Iakunchykova OP, Andreeva TI, Nordstrom DL, et al. The impact of early life stress on risk of tobacco smoking initiation by adolescents. Addict Behav. 2015;50:222-228. doi:10.1016/j.addbeh.2015.06.014

17. Kennedy B, Chen R, Fang F, et al. Low stress resilience in late adolescence and risk of smoking, high alcohol consumption and drug use later in life. J Epidemiol Community Health. 2019;73(6):496-501. doi:10.1136/jech-2018-211815

18. Stubbs B, Veronese N, Vancampfort D, et al. Perceived stress and smoking across 41 countries: A global perspective across Europe, Africa, Asia and the Americas. Sci Rep. 2017;7(1):7597. doi:10.1038/s41598-017-07579-w

19. Kearns NT, Carl E, Stein AT, et al. Posttraumatic stress disorder and cigarette smoking: A systematic review. Depress Anxiety. 2018;35(11):1056-1072. doi:10.1002/da.22828

20. Fidler JA, West R. Self-perceived smoking motives and their correlates in a general population sample. Nicotine Tob Res. 2009;11(10):1182-1188. doi:10.1093/ntr/ntp120

21. Buchmann AF, Laucht M, Schmid B, Wiedemann K, Mann K, Zimmermann US. Cigarette craving increases after a psychosocial stress test and is related to cortisol stress response but not to dependence scores in daily smokers. J Psychopharmacol. 2010;24(2):247-255. doi:10.1177/0269881108095716

22. Perkins KA, Grobe JE. Increased desire to smoke during acute stress. Br J Addict. 1992;87(7):1037-1040. doi:10.1111/j.1360-0443.1992.tb03121.x

23. Yu G, Chen H, Sharp BM. Amplified reacquisition of nicotine self-administration in rats by repeated stress during abstinence. Psychopharmacology (Berl). 2014;231(16):3189-3195. doi:10.1007/s00213-014-3501-x

24. Parnia A, Siddiqi A. Socioeconomic disparities in smoking are partially explained by chronic financial stress: marginal structural model of older US adults. J Epidemiol Community Health. 2020;74(3):248-254. doi:10.1136/jech-2019-213357

25. Welch AE, Jasek JP, Caramanica K, Chiles MC, Johns M. Cigarette smoking and 9/11-related posttraumatic stress disorder among World Trade Center Health Registry enrollees, 2003-12. Prev Med. 2015;73:94-99. doi:10.1016/j.ypmed.2015.01.023

26. Cambron C, Haslam AK, Baucom BRW, et al. Momentary precipitants connecting stress and smoking lapse during a quit attempt. Health Psychol. 2019;38(12):1049-1058. doi:10.1037/hea0000797

27. Jackson KJ, Muldoon PP, De Biasi M, Damaj MI. New mechanisms and perspectives in nicotine withdrawal. Neuropharmacology. 2015;96(Pt B ):223-234. 
doi:10.1016/j.neuropharm.2014.11.009

28. Fluharty M, Taylor AE, Grabski M, Munafò MR. The Association of Cigarette Smoking With Depression and Anxiety: A Systematic Review. Nicotine Tob Res. 2017;19(1):3-13. doi:10.1093/ntr/ntw140

29. Hajek P, Taylor T, McRobbie H. The effect of stopping smoking on perceived stress levels. Addiction. 2010;105(8):14661471. doi:10.1111/j.1360-0443.2010.02979.x

30. Taylor G, McNeill A, Girling A, Farley A, Lindson-Hawley $\mathrm{N}$, Aveyard P. Change in mental health after smoking cessation: systematic review and meta-analysis. BMJ. 2014;348:g1151. doi:10.1136/bmj.g1151

31. Kim SJ, Chae W, Park WH, Park MH, Park EC, Jang SI. The impact of smoking cessation attempts on stress levels. BMC Public Health. 2019;19(1):267. doi:10.1186/s12889-019-6592-9

32. Kouvonen A, Kivimäki M, Virtanen M, Pentti J, Vahtera J. Work stress, smoking status, and smoking intensity: an observational study of 46,190 employees. J Epidemiol Community Health. 2005;59(1):63-69. doi:10.1136/jech.2004.019752

33. Nielsen MG, Ørnbøl E, Vestergaard M, et al. The construct validity of the Perceived Stress Scale. J Psychosom Res. 2016;84:22-30. doi:10.1016/j.jpsychores.2016.03.009

34. Cambron C, Hopkins P, Burningham C, Lam C, Cinciripini $\mathrm{P}$, Wetter DW. Socioeconomic status, mindfulness, and momentary associations between stress and smoking lapse during a quit attempt. Drug Alcohol Depend. 2020;209:107840. doi:10.1016/j.drugalcdep.2020.107840

\section{CONFLICTS OF INTEREST}

The authors have each completed and submitted an ICMJE form for disclosure of potential conflicts of interest. The authors declare that they have no competing interests, financial or otherwise, related to the current work. All authors report funding by The Health Foundation, Denmark (grant ID 18-B-0355). C. Pisinger also reports grants from Danish Health Authorities: update of national smoking cessation guidelines, and other funds from European Respiratory Society conference (2018 and 2019 invited speaker) and annual meetings, as head of the tobacco control group in the ERS.

\section{FUNDING}

This study was funded (grant ID 18-B-0355) by The Health Foundation, Denmark (an independent charity that funds research and development related to health and social issues) and the Danish Heart Foundation partly funded the salary of C. Pisinger during work on this study. The funders were not involved in the choice of research or any step of this study, and will first be presented with the study when it is published.

\section{AUTHORS' CONTRIBUTIONS}

All authors discussed the study design and data interpretation, contributed to subsequent versions of the manuscript and read and approved the final manuscript. LPB wrote the first draft of the manuscript together with CP. AHA wrote the statistics section and prepared and analyzed the data and discussed the results with LPB and CP. All authors read and approved the final version of the manuscript.

PROVENANCE AND PEER REVIEW

Not commissioned; externally peer reviewed. 